Cahiers de philosophie de l'université de

CAHIERS DE PHILOSOPHIE
DE L L UNIVERSTIE DE CAEN

Caen

48 | 2011

Philosophie russe

\title{
Temporalité et eschatologie en Russie. L'exemple de Nicolas Berdiaev
}

\section{Maryse Dennes}

\section{(2) OpenEdition}

1 Journals

Édition électronique

URL : https://journals.openedition.org/cpuc/987

DOI : $10.4000 /$ cpuc. 987

ISSN : 2677-6529

Éditeur

Presses universitaires de Caen

\section{Édition imprimée}

Date de publication : 31 octobre 2011

Pagination : 111-121

ISBN : 978-2-84133-385-1

ISSN : 1282-6545

\section{Référence électronique}

Maryse Dennes, «Temporalité et eschatologie en Russie. L'exemple de Nicolas Berdiaev », Cahiers de philosophie de l'université de Caen [En ligne], 48| 2011, mis en ligne le 01 septembre 2020, consulté le 07 février 2023. URL : http://journals.openedition.org/cpuc/987 ; DOI : https://doi.org/10.4000/cpuc. 987

\section{(c) (†) 8}

Creative Commons - Attribution - Pas d'Utilisation Commerciale 4.0 International - CC BY-NC 4.0

https://creativecommons.org/licenses/by-nc/4.0/ 


\title{
Temporalité et eschatologie en Russie. L'exemple de Nicolas Berdiaev
}

\begin{abstract}
Reconnaître chaque fois les conditions de réalisation d'une histoire enracinée dans l'expérience humaine et dans la capacité humaine de donner sens, tel est le destin commun de l'Occident et de la Russie. Au sein de leur différence les deux s'interpellent. Ils se nécessitent même dans leur complémentarité comme une condition essentielle, à notre époque, du maintien de l'homme dans l'horizon de l'être.

N. Berdiaev, Essai de métaphysique eschatologique.
\end{abstract}

$\mathrm{L}$

A QUESTION DE LA TEMPORALITÉ est une problématique philosophique qui a été particulièrement développée au cours du $\mathrm{XX}^{\mathrm{e}}$ siècle, et, plus particulièrement, dans le contexte de la phénoménologie, sur la base d'une interprétation critique de l'idéalisme allemand. Le philosophe le plus représentatif d'une telle démarche est Martin Heidegger. C'est sur lui que nous nous appuierons tout d'abord pour montrer comment on a pu parler d'une temporalité spécifique de l'histoire de l'Occident et comment, sur des bases ontologiques analogues, faisant intervenir un certain rapport de l'être et de l'étant, peut être pensé un mode de «temporalisation» qui serait posé comme spécifique de la Russie. L'exemple de Nicolas Berdiaev nous permettra de montrer ensuite et de façon privilégiée comment l'exercice de la pensée peut lui-même engendrer un mode spécifique de la totalisation de l'étant, et peut se trouver ainsi en correspondance avec un mode de temporalité inhérent à l'histoire dont cette pensée participe.

Dans nombre de ses écrits - à commencer par Être et temps ${ }^{1}$ et jusqu'à Temps et être ${ }^{2}$, qui fait subir un renversement de perspective à l'histoire

1. M. Heidegger, Être et temps, Paris, Gallimard, 1986 (trad. par F. Vezin); Paris, Authentica, 1985 (trad. par E. Martineau).

2. M. Heidegger, Questions 4, Temps et être, Paris, Gallimard, 1976 (trad. par F. Fédier, J. Beaufret), p. 9-106. 
de l'être -, Heidegger se réfère aux représentants de l'idéalisme allemand pour montrer comment apparaît, à partir de leur époque, un rapport spécifique à l'histoire, fondé sur l'idée que la Raison est, par elle-même, susceptible de rassembler tous les éléments du monde extérieur et donc de leur donner sens. C'est cette donation de sens qui est révélatrice d'un mode d'organisation des événements dans le temps. Elle les «temporalise »3; elle est, de ce fait, constitutive d'une "temporalité " ${ }^{4}$ ainsi que d'un mode d'être dans l'histoire qui est nommé "historialité»5.

C'est dans son article intitulé "Identité et différence ${ }^{6}$ que les bases de cette démarche se trouvent clairement exposées. Par l'intermédiaire de Hegel, Heidegger renvoie à Parménide afin de mettre en valeur cette identité de l'acte et de l'objet de la pensée, à partir de laquelle peut être engagée une interprétation de l'histoire. «Il a fallu [écrit-il] à la pensée plus de deux mille ans pour dégager et comprendre une relation aussi simple que la médiation intérieure à l'identité» 7 . La "médiation", c'est précisément cette aptitude qu'a la pensée elle-même - à partir de son identité à l'être - de se rapporter aux éléments extérieurs, aux faits et aux événements, pour les rassembler, pour leur permettre de signifier; c'est aussi cette façon qu'elle a de renouveler cet acte de rassemblement selon des paradigmes nouveaux, et de déterminer ainsi plusieurs étapes dans l'histoire de son propre devenir. «La pensée (das Denken) [nous dit-il], parce qu'elle fait corps avec l'être - vise l'être comme fond (Grund)»; or le «fond", c'est le «Logos», entendu comme ce qui rassemble et laisse étendu-devant: $1^{\prime}$ «Hen Panta» ${ }^{8}$. Nous sommes ici placés dans la perspective d'une logique de l'être, dont la fonction est de déceler le mode fondamental du rassemblement de l'étant et les différentes étapes de la manifestation de ce mode de rassemblement. «Hegel [nous dit-il encore], fidèle à la tradition, découvre le propos de la pensée dans l'étant comme tel et dans son tout, dans le mouvement de la pensée qui la conduit de sa vacuité à l'épanouissement de sa plénitude » ${ }^{9}$. Pour résumer, nous pouvons dire: une histoire prend forme parce que se trouve fondée, en l'homme, la possibilité de signifier, la possibilité de rassembler et de projeter, la possibilité aussi de passer de l'une à l'autre des formes possibles du rassemblement et d'engendrer

3. Sur l'emploi de ce terme, voir M. Heidegger, Etre et temps (trad. par E. Martineau), deuxième section: «Dasein et temporalité» et, en particulier, chapitre V: «Temporalité et historialité», p. 295.

4. Ibid.

5. Ibid.

6. M. Heidegger, «Identité et différence», in Questions 1, Paris, Gallimard, 1968 (trad. par A. Préau), p. 253-310.

7. Ibid., p. 275.

8. Ibid., p. 291-292.

9. Ibid., p. 291. 
ainsi un devenir propre à l'homme - ce que nous pouvons nommer, avec Heidegger, une «temporalisation de la temporalité» ${ }^{10}$.

Or, le but de notre travail est de montrer que c'est en plusieurs modes que peut se «temporaliser» la temporalité, que c'est en diverses façons que peut être pensé le rapport de l'Un au Tout, et que, dans le surgissement de l'être par l'identité à soi de la pensée, il y a plusieurs façons possibles de rapporter toute chose à cette identité; en un mot: qu'il y a, pour l'homme, plusieurs modalités d'être au monde et dans l'histoire ${ }^{11}$.

Pour cela, à côté de Heidegger, nous prendrons en compte une autre réflexion sur l'histoire, qui se trouve développée à la même époque. En 1923, Berdiaev écrit, dans Le sens de l'histoire: "Il faut que non seulement l'objet soit historique, mais que le sujet le soit aussi pour que celui qui recherche la connaissance historique sente en soi et découvre en soi ce qui est historique ${ }^{12}$. Pour l'un comme pour l'autre, pour Berdiaev comme pour Heidegger, l'histoire doit être envisagée dans le rapport qu'elle entretient avec le sujet qui la pense. C'est le sujet qui donne à l'histoire son mode de développement. C'est lui qui est le révélateur et le producteur du mode selon lequel se déploie la temporalité.

Dans l'un et l'autre cas, il y a référence (implicite ou non) au principe parménidéen de l'identité de l'être et de la pensée. Dans l'un et l'autre cas, ce principe est posé comme la condition du rassemblement de l'étant. Cependant, Berdiaev témoigne simultanément d'une autre tradition culturelle, d'un autre mode d'exercice de la pensée et donc, sans aucun doute, d'une autre façon de se rapporter à l'étant et de le totaliser. Pour le comprendre, il faut tout d'abord nous attacher à expliquer comment il peut y avoir une forme de temporalité où les éléments du monde extérieur - faits ou événements, par exemple - se trouvent rassemblés non point en vue de connaître, de maîtrise ou de dominer, mais, du fait simplement qu'il y a une visée, une tentative de nommer, quelque chose qui n'est rien d'étant (qui n'est pas du monde). Il s'ensuivra une prise en compte du rapport que le temps entretient avec l'eschatologie, et cela nous apparaîtra comme spécifique d'un mode de temporalité.

10. Voir M. Heidegger, Etre et temps (trad. par E. Martineau), p. 295.

11. Cette problématique constitue le thème principal de ma thèse Dualisme et principe de totalité. Recherche d'un fondement de la différence entre Russie et Occident, t. I, II, soutenue à l'université de Paris X - Nanterre en 1990, enregistrée au fichier central des thèses sous le numéro $8503341 \mathrm{~L}$. À ce sujet, voir aussi M. Dennes, Russie-Occident, philosophie d'une différence, Paris, Mentha, 1991.

12. N. Berdiaev, Le sens de l'histoire - Essai d'une philosophie de la destinée humaine, Paris, Aubier, 1949 (trad. par S. Jankelevitch), p. 30 [traduction de Smysl istorii. Opyt filosofii čeloveceskoj sud'by, Paris, YMCA-Press, 1969]. 
À un niveau théorique et en adoptant l'attitude phénoménologique de la description des essences, nous partons du fait que l'étant peut être rassemblé de façons différentes selon que le regard de l'homme - sujet transcendantal enraciné dans l'identité de l'être et de la pensée, prend ou non en compte la réalité qui lui est opposée. Si cette réalité est principalement ignorée, nous admettons l'existence d'un regard originellement tourné vers ce qui n'est d'aucun monde - attitude primordiale de l'expérience mystique qui implique néanmoins, mais secondairement, un type de rapport au monde, et donc une logique, dont les données fondamentales peuvent être constitutives d'une temporalité.

Nous ne retiendrons ici que les points essentiels permettant d'avancer vers la constitution d'une telle temporalité:

D’une part, du fait que nous sommes placés sous le signe de l'identité, ce qui se trouve visé et qui est en soi indicible, infini, illimité, définissable uniquement apophatiquement, se trouve, du fait même de la visée, rassemblé sous une forme qui équivaut nécessairement à la totalisation complète de tous les éléments du monde extérieur. Vivre et dire cette expérience, c'est donc la fixer en ce qu'elle n'est pas, en son contraire absolu. C'est poser une réalité qui s'oppose totalement à ce qui l'a initialement provoquée. C'est expérimenter une "épreuve du tout »; c'est constater un «dualisme absolu»; c'est installer un monde absolument totalisé; ce n'est pourtant pas encore s'installer dans une temporalité, ni dans le mode de temporalité qui a été relevé par Heidegger comme caractéristique de l'histoire de l'Occident. Ce n'est pas non plus s'installer dans un autre mode de temporalité, puisque, d'une part, ce rassemblement est immédiatement le plus important qui puisse être pensé et qu'il ne peut donc en aucune façon faire place à une forme qui lui serait différente ou supérieure, et puisque, d'autre part, le phénomène qui est ici constaté est celui d'une fixation et non point celui d'une ouverture exigée par le lancement d'un processus historial. Nous pouvons dire qu'ici se produit un passage de l'expérience vécue à sa fixation mondaine, mais que, sur cette base, il n'y a, pour l'instant, aucun changement pensable et donc apparemment aucun fondement possible de temporalité.

Pourtant si, à ce niveau théorique et eidétique, nous ajoutons une situation particulière, celle d'une confrontation de ce regard initial avec un autre regard qui se trouve principalement dirigé vers ce qui lui est ou peut lui être opposé, alors ce deuxième regard, proche du premier du fait de son enracinement dans la même identité de l'être et de la pensée, mais différent de lui néanmoins par sa direction initiale, ne retient et ne connaît du premier que ce qui a pris (ou prend) forme dans le monde. C'est la présence de ce point de vue qui provoque, face à l'épreuve de fixation, une «remise en mouvement». 
À cause de cet «autre» dorénavant présent au sein de l'expérience décrite, un changement d'attitude se trouve nécessité. Face à un regard qui, par son unilatéralité, fausse ce qui est dit sur la vision initiale, il s'agit de tenter de dire de nouveau ce qui était initialement visé, de justifier son mode de présence au monde en prouvant sa provenance. Face à l'inefficacité des preuves, il s'agit de préserver son authenticité et d'expérimenter de nouveau l'initial. Abandonner la démonstration pour en venir à la description et, à travers ce dire, se retrouver renvoyé, de l'intérieur du monde, à une expérience qui nie le monde objectivé, à une adhésion à soi originelle - telle est la base phénoménologique qui nous permet de parler d'un fondement de temporalité.

En effet, nous accédons ici à un mouvement de fond qui peut être pensé comme sous-jacent à une histoire de l'homme. D'une part, du fait de l'expérience initialement vécue, à cause du dire inhérent à cette expérience, la totalité du monde se trouve projetée, objectivée, mais d'autre part, à cause de l'inefficacité des preuves, du fait de la visée de cette expérience initiale, cette totalité se trouve rejetée, dépassée pour permettre le dire d'une expérience qui la nie et la provoque simultanément. Un va-et-vient s'installe entre la «preuve» et le «dire» de l'expérience originelle, entre le projet et le rejet de la totalité de l'étant. Cependant, une chose reste encore à souligner: pour «exister (ek-sister) $»^{13}$ de cette façon dans le monde, pour fonder, de cette façon, une histoire, l'homme jamais ne se situe complètement au sein du monde ainsi projeté et objectivé. Le domaine de son «ek-sistence $»^{14}$ n'est $^{\prime}$ ni totalement hors du monde, ni totalement dans le monde. Il est plutôt un «entre-deux» parcouru dans un sens et dans l'autre, une position transcendantale, un espace «existential ${ }^{15}$, susceptible de fonder une histoire, et qui

13. Sur l'emploi de ce terme, voir M. Heidegger, Lettre sur l'humanisme, in Questions 3, Paris, Gallimard, 1966 (trad. par R. Munier), p. 71-157, et, en particulier, p. 92-93, lorsque M. Heidegger commente la phrase de Sein und Zeit (Être et temps), «L'essence de l'être-là [Dasein] réside dans son existence»: «Il ne s'agit pas là d'une opposition entre existentia et essentia, car ces deux déterminations de l'Être en général, et à bien plus forte raison leur rapport, ne sont pas en question [...]. Bien plutôt veut-elle [la phrase] dire que l'homme déploie son essence de telle sorte qu'il est le "là" [das Da], c'est-à-dire l'éclaircie de l'Être. Cet "être" du là et lui seul, comporte le trait fondamental de l'ek-sistence, c'est-à-dire de l'ins-tance extatique dans la vérité de l'Être».

14. Ibid., p. 93: «L'essence extatique de l'homme repose dans l'ek-sistence, qui reste distincte de l'existentia pensée d'un point de vue métaphysique». Et aussi, p. 95: «Dans son contenu, l'ek-sistence signifie ex-tase [hinaus-stehen] en vue de la vérité de l'Être [...]. La proposition: "l'homme ek-siste" n'est pas une réponse à la question de savoir si l'homme est réel ou non; elle est une réponse à la question portant sur l'“essence" de l'homme».

15. Sur l'emploi du mot «existential», en tant qu'il se trouve réservé pour désigner ce qui se rapporte à l'essence de l'être-là (Dasein), voir M. Heidegger, «Avant-propos de H. Corbin », in Questions 1, p. 14. 
se détermine par l'alternance du «dire» et de la "preuve» d'une expérience originelle d'adhésion à soi, par l'alternance aussi, sans cesse renouvelée, de ce que nous pouvons appeler une «forme réelle» et une «forme dérivée de l'eschatologie». Le temps proprement humain, le temps historial ${ }^{16}$, n'est alors ni totalement ouvert sur l'éternité, ni totalement absorbé par l'adhésion au monde extérieur (ou par la connaissance de ce monde). Il est la forme constante d'une oscillation entre deux domaines qui sont, chacun à leur façon, ceux d'un "eskhaton", ceux d'une fin des temps. Mais alors que la quête d'éternité correspond à la visée d'une « eschatologie réelle », la forme que prend le monde totalisé n'est qu'une image, détériorée, de ce qui est recherché. C'est elle que nous appelons "forme dérivée de l'eschatologie", c'est-à-dire forme objectivée, secondaire d'une eschatologie plus essentielle et primordiale. En ce sens, cette forme dérivée ne peut qu'être génératrice d'illusions: illusion d'existence autant qu'illusion d'éternité.

La mise en valeur d'une telle forme de temporalité, basée sur l'oscillation entre une forme réelle et une forme dérivée de l'eschatologie, et dépendante aussi de deux modes différents de se rapporter à l'être par le langage (la «preuve» et le «dire»), permet deux choses:

- D’une part, de saisir le mouvement de fond de l'histoire de la Russie et de comprendre aussi le risque inhérent à cette façon d'être dans l'histoire ce qui, de tout temps, a menacé la Russie dans son existence: la croyance millénariste en l'avènement possible d'un succédané du Royaume de Dieu sur la terre, ou bien, de façon plus générale, l'absorption d'une réalité existentiale par la forme que peut prendre la totalité du monde; et, à côté de cela, par moments, par réaction, le rejet brusque de toute forme établie, l'abandon au chaos.

- D'autre part, elle permet de comprendre, d'une façon nouvelle, le sens et la portée du discours souvent antinomique des penseurs russes. Ils sont nombreux à avoir revécu, par la forme de leur pensée, le mouvement de fond qui traverse aussi l'histoire de la Russie. Nicolas Berdiaev en est un exemple privilégié. Ressentant l'originalité de l'histoire de la Russie par rapport à celle de l'Occident, ces penseurs cherchaient à en saisir le sens, mais ne parvenaient pas à en dégager les structures transcendantales. Immergés dans la réalité existentielle de leur culture, ils décrivaient, à travers les événements, la contradiction qu'ils éprouvaient fondamentalement, mais c'est plus analogiquement que logiquement qu'ils témoignaient, dans leur pensée, de leur adhésion à une spécificité historiale.

16. M. Heidegger, «Avant-propos de H. Corbin», p. 16: «Sa désinence offre l'avantage de l'appeler du côté de l'existential». 
Le choix du sujet ne me permet pas d'aborder en détail le premier point. Le passage du niveau théorique et eidétique au niveau purement historique est très délicat. Il nécessite d'abord, au niveau transcendantal, l'élaboration d'un système de catégories permettant de différencier les modes possibles de totalisation de l'étant et de déterminer, sur cette base, un horizon d'historicité différent de celui de l'Occident. Il nécessite aussi, à un niveau purement historique, une étude détaillée des événements et des mouvements culturels, des influences subies aux différentes époques et des façons de réagir à ces influences. Enfin, le rapport de l'historique à l'historial ne peut être établi qu'avec une grande prudence, en insistant sur le fait que l'objectivité du schéma proposé dépend de son aptitude à être confronté à d'autres schémas interprétatifs et que sa portée heuristique ne peut être qu'à la mesure de son caractère opératoire, autant dans le domaine de la philosophie générale (repositionnement de la question ontologique) que dans celui de la philosophie de l'histoire (recherche d'une spécificité de l'histoire de la Russie par rapport à celle de l'Occident).

Ces différents aspects ont déjà fait l'objet de divers travaux ${ }^{17}$.

Je ne me consacrerai donc ici qu'à une illustration, celle que peuvent nous fournir les écrits de Berdiaev. Et plus particulièrement, à la lumière de ce qui vient d'être dit sur la temporalité et l'eschatologie, nous essaierons de comprendre la raison de son discours antinomique sur le temps. L'antinomie peut être relevée dans son essai intitulé Cinq méditations sur l'existence ${ }^{18}$.

D’une part, Berdiaev affirme: «Tout ce qui n'est pas éternel, tout ce qui n'a pas l'éternité pour origine et pour fin est dépourvu de toute valeur et destiné à disparaître» (p. 159), et d'autre part, il écrit, quelques pages plus loin: «Le destin de l'existence humaine s'accomplit dans le temps, est placé sous le signe du temps» (p. 133). Puis, il propose lui-même une explication et une synthèse de ces deux énoncés apparemment contradictoires: «L'évolution se déploie dans le temps et est subordonnée au temps. Mais les actes premiers de l'esprit engendrent le temps lui-même [...]. Le temps est en quelque sorte une chute hors de l'éternité et, d'autre part, le temps est intérieur à l'éternité » (p. 148). Il conclut finalement de la façon suivante: «Le problème du temps devient finalement le problème de l'eschatologie» (p. 152).

À travers ces quelques exemples, nous voyons comment Berdiaev énonce sa pensée et comment il tente de décrire une expérience spécifique du temps.

17. Voir note 11

18. N. Berdiaev, Cinq méditations sur l'existence. Solitude, société et communauté, Paris, Aubier, 1936 (trad. par I. Vildé-Lot) [traduction de Ja i mir ob"ektov (Le Moi et le monde des objets)]. Les références de pages qui suivent renvoient à cet ouvrage. 
Or, si nous nous plaçons du point de vue d'un mode de temporalité susceptible de se manifester dans l'histoire de la Russie, nous avons à notre disposition une logique du devenir qui nous permet d'envisager de nouveau les énoncés de Berdiaev et d'en dépasser le caractère apparemment contradictoire.

En un premier temps, Berdiaev ne parle pas d'une quelconque opposition entre l'homme et le monde. Ce qui importe, au contraire, pour lui, c'est de relever la situation première de l'homme par rapport à lui-même. Il écrit: «Ce qui est premier, ce n'est pas comme le pensent beaucoup de philosophes, la conscience. C'est le moi immergé dans l'existence» (p. 94). Mais, en un deuxième temps, du fait qu'il pense le moi et tout ce qui constitue le moi à travers un "acte créateur synthétique ${ }^{19}$, Berdiaev tire immédiatement certaines conséquences de son premier énoncé. D’une part, par rapport à la totalité du monde, il écrit: «Originellement le moi et le tout, le tout et le moi ne se distinguent pas» (p. 94). D'autre part, par rapport au temps qui n'est pas considéré comme le domaine de ce qui a été initialement expérimenté il précise: "L'existence humaine n'est dans le temps qu’à la suite d'une chute, et elle doit sortir du temps» (p. 160).

Si l'on suit la pensée de Berdiaev sur l'être et sur le moi, il y a, dès les premiers énoncés, une incertitude et une ambiguïté. Une apparente contradiction se laisse percevoir qui oppose un monisme absolu et un dualisme radical. Lui-même, dans Le sens de la création ${ }^{20}$, énonce cette situation de la façon suivante: «Je confesse un dualisme manichéen. Qu'il en soit ainsi. Le monde est le mal [...]. Il faut sortir de ce monde, le surmonter jusqu'à la fin» (p. 36). Et il ajoute: "Cette antinomie du dualisme et du monisme, elle m'est connue jusqu'au bout et je l'accepte comme inévitable dans la conscience» (p. 37).

Cependant, malgré ce désir si clairement exprimé, Berdiaev ne s'est jamais départi de la situation de l'homme dans le monde. Nous pouvons même dire que c'est à partir d'une telle situation qu'il envisageait tous les problèmes. C'est donc qu'il prenait un point de vue déjà second par rapport à l'expérience originelle et fondatrice, initialement évoquée. Par son expérience propre de pensée, il manifestait un mode d'être spécifique qui nous renvoie aux conditions que nous avons déterminées comme étant celles d'un mode, lui aussi spécifique, de temporalité: d'une part, Berdiaev se plaçait dans la

19. Voir à ce sujet, N. Berdiaev, Essai de métaphysique eschatologique; acte créateur et objectivation, Paris, Aubier, 1946, p. 198: «Non seulement ce qui crée le moi mais l'existence même du moi est un acte créateur synthétique».

20. N. Berdiaev, Le sens de la création. Un essai de justification de l'homme, Paris, Desclée de Brouwer, 1955 (trad. par L. Julien-Cain) [traduction de Smysl tvorčestva. Opyt opravdanija čeloveka [1915], Moscou, Paris, YMCA-Press, 1991]. 
position de celui qui vise un au-delà de tout monde; mais d'autre part, tentant de dire ce qui était visé, il se retrouvait lui-même toujours engagé dans une épreuve qui était celle de la présence contraignante du monde objectivé dans sa totalité. D'une part, il cherchait à décrire la situation primordiale du moi par rapport à lui-même; mais d'autre part, il ne pouvait le faire qu'à travers des énoncés qui apparaissaient comme contradictoires. Finalement, Berdiaev se trouvait dans une situation où, expérimentant simultanément les deux faces d'une même expérience, il les disait aussi à travers une opposition qui se trouvait, à son tour, ressentie comme originelle. Cela, me semble$\mathrm{t}$-il, nous permet de comprendre le caractère antinomique de la pensée de Berdiaev. En tant que penseur, il expérimentait, plus qu'il n'analysait, un fondement existential qu'il tentait de décrire, et cette description était, à son tour, productrice d'une expérience qui le mettait à distance de la situation d'origine. En fait, ce qu'il éprouvait, au niveau de son expérience personnelle, par l'intermédiaire de la prise de conscience qu'il tentait d'en faire, n'était qu'une reproduction analogique, une reconnaissance par analogie, d'une modalité d'être susceptible d'être pensée comme fondamentale pour la compréhension de l'histoire de la Russie.

C'est ainsi que, dans Le sens de l'histoire, il écrivit: «Aller dans la profondeur du temps, c'est aller dans la profondeur de soi-même. Seulement dans la profondeur de soi-même, l'homme peut trouver réellement la profondeur du temps ${ }^{21}$.

Cette expérience du temps est identifiée, par Berdiaev, à l'expérience chrétienne. Dans un tel contexte, l'au-delà du temps est assimilé au Royaume de Dieu et c'est parce qu'il y a, à partir des données de l'expérience humaine, visée du Royaume de Dieu que cet «au-delà du temps» est pensé comme une fin des temps, comme un eskhaton. Dans son ouvrage intitulé De l'inégalité, il déclare: «L'avènement du Christ n'a pas été l'avènement du Royaume de Dieu sur la terre, dans le monde matériel. Il n’a été que la promesse du Royaume du Dieu. Le Christ enseignait que son Royaume n'est pas de ce monde. Et ce monde ne peut pas contenir son Royaume ${ }^{22}$.

D'une part, il y a donc, pour le penseur russe, une vraie eschatologie, celle qui est basée sur un rejet de l'objectivation, c'est-à-dire, nous dit-il encore, "de ce qui est étranger ${ }^{23}$; mais d'autre part, cette vraie eschatologie est productrice d'une eschatologie dérivée, d'une fausse eschatologie, celle qui consisterait à fixer dans le monde et dans le temps ce qui est visé comme

21. N. Berdiaev, Le sens de l'histoire..., p. 30.

22. N. Berdiaev, De l'inégalité, Lausanne, l'Âge d'homme, 1976 (trad. par C. et A. Andronikof), p. 229.

23. N. Berdiaev, Cinq méditations sur l'existence..., p. 52. 
étant au-delà du monde et au-delà du temps. C'est dans cette alternance d'une vraie et d'une fausse eschatologie, dans cette possibilité de passer de l'une à l'autre, qu'un mode spécifique de temporalité se concrétise. Dans les Cinq méditations sur l'existence, Berdiaev écrit à ce sujet: «Le temps est un changement dans deux sens différents: dans le sens de l'élévation de la vie et dans le sens de la mort ${ }^{24}$.

Dans son œuvre, la création inhérente à l'activité humaine est souvent invoquée comme ce qui manifeste le mieux ce mode d'être par rapport au temps, qui est aussi fondateur d'un mode de temporalité.

Dans l'acte créateur de l'esprit [écrit-il encore], l'homme sort de la subjectivité close par deux voies: celle de l'objectivation et celle de la transcendance. Par la voie de l'objectivation, l'acte créateur s'adapte à l'état de ce monde et n'atteint pas son état final: il est interrompu. Par la voie de la transcendance, l'acte créateur perce jusqu’à la réalité nouménale et s'achemine vers la transfiguration finale du monde ${ }^{25}$.

Dans le domaine de l'art, comme dans celui de l'histoire, dans tout ce qui concerne les résultats de l'action proprement et uniquement humaine, il y a, pour Berdiaev, risque d'échapper à l'authenticité de la réalisation de l'homme, risque de sortir des conditions imparties à cette réalisation. Ce qui se trouve objectivé (œuvre d'art, structure sociale ou politique) a besoin d'être spontanément nié, rejeté sous cette forme, pour être restauré dans sa signification humaine.

La fin des temps elle-même, en tant qu'intérieure à l'expérience personnelle, subit, chez Berdiaev, cette double interprétation, cette brisure inhérente à l'expérience de toute réalité existentielle, dès lors qu'on veut exprimer cette réalité et qu'on la met en rapport avec le monde objectivé. C'est pour cette raison que, dans cette approche du temps et de l'eschatologie, comme dans toute approche faite par Berdiaev, il ne faut pas retenir l'usage des antinomies comme le signe d'un manque de rigueur philosophique. Cet usage doit être vu, au contraire, comme l'émanation d'une logique spécifique de l'expérience humaine, d'une logique apte à déterminer une histoire. D'un côté Berdiaev voit l'homme engagé dans le temps, irrévocablement lié à un monde objectivé, et c'est pour cette raison qu'il pense alors la fin des temps comme identique à une fixation dans la totalité du monde créé. C'est pour cette raison qu'il envisage aussi cet avènement comme celui d'un troisième âge de l'humanité et qu'il développe une forme de millénarisme. Mais d'un autre côté, Berdiaev voit l'homme déjà transfiguré, dans sa situation originelle

24. N. Berdiaev, Cinq méditations sur l'existence..., p. 135.

25. N. Berdiaev, Essai de métaphysique eschatologique..., p. 22. 
de simple adhésion à soi, hors du monde et hors du temps, et c'est pour cette raison qu'il annonce alors l'âge de la création non plus comme une époque à venir, mais comme un autre niveau d'existence, perceptible par un regard qui parvient à viser l'au-delà du temps, comme un présent déjà là et éternel. Dans la perspective chrétienne qui est la sienne, l'homme est pensé, à travers l'espoir du salut, comme pouvant être toujours et simultanément encore dans le temps et déjà dans l'éternité. Nous pouvons dire qu'il est engagé dans une forme de temporalité qui a partie liée avec l'eschatologie.

Dans son ouvrage De l'inégalité, Berdiaev réunit les deux positions et insiste sur leur caractère indissociable. Il écrit:

Le plan apocalyptique auquel nous renvoyons l'avènement de la fin du monde, la solution de l'histoire universelle, ne peut être conçu ni d'une manière totalement immanente, ni complètement transcendante, ni exclusivement d'ici-bas, ni uniquement dans l'au-delà. C'est le problème de la relation entre le temps et l'éternité qui est antinomique ${ }^{26}$.

Finalement, comme nous l'avons pressenti à plusieurs reprises, la contradiction n'est pas dépassée, elle est réaffirmée. Le dualisme n'est pas rejeté, il est proclamé comme incontournable parce qu'inhérent à un type bien précis de l'expérience humaine. La pensée de Berdiaev, fidèle à sa propre expérience, n'analyse pas le mode d'existence qu'elle expérimente. Elle tente seulement de le dire et, de ce fait, elle le reproduit. Elle ne dégage pas des transcendantaux. Pour dire l'expérience, elle la revit. Pour justifier les oppositions, elle les recrée, elle les laisse surgir de l'expérience de la vie. Mais en cela précisément nous pouvons dire que la pensée de Berdiaev illustre de façon privilégiée ce que nous avons tenté tout d'abord de dire sur la temporalité, l'eschatologie et la Russie.

Maryse Dennes

Université Michel de Montaigne - Bordeaux 3

26. N. Berdiaev, De l'inégalité, p. 230. 